\title{
Rate Dependencies and Energy Absorption Characteristics of Nanoreinforced, Biofiber, and Microcellular Polymer Composites
}

\author{
Alan Argento, ${ }^{1}$ Wonsuk Kim, ${ }^{1}$ Ellen C. Lee, ${ }^{2}$ Angela M. Harris, ${ }^{2}$ Deborah F. Mielewski ${ }^{2}$ \\ ${ }^{1}$ Department of Mechanical Engineering, The University of Michigan-Dearborn, Dearborn, Michigan 48128 \\ ${ }^{2}$ Materials Research and Advanced Engineering Department, Research and Innovation Center, \\ Ford Motor Company, Dearborn, Michigan 48124
}

\begin{abstract}
The effects of loading rate on bio-, nano-, and microcellular composite systems have been studied. Fiberresin systems have been manufactured and dynamically tested at various speeds to assess their strainrate dependencies (rate hardening) and energy-dissipation characteristics compared to conventional materials. The following composite systems have been fabricated and studied: polypropylene/sisal fiber biocomposite, hemp/vinyl ester biocomposite, thermoplastic olefin/nanoclay composite, microcellular polypropylene/sisal fiber biocomposite, and microcellular thermoplastic olefin/nanoclay composite. It has been determined that the biocomposite systems studied possess unique energy dissipation characteristics and muted rate dependence, while the nanocomposite system did not. In addition, microcellular foaming of these materials further enhanced the effects. Though the exact mechanisms at play are not fully understood at this point, it has been found that in addition to the microcellular voids, the anatomical vasculature of the natural fibers may play a role in energy dissipation processes in these hybrid materials. POLYM. COMPOS., 32:14231429, 2011. () 2011 Society of Plastics Engineers
\end{abstract}

\section{INTRODUCTION}

Interest in sustainable and natural materials as replacements for petroleum based materials in engineering settings is growing due to environmental benefits and potentially lower cost. One technology of recent interest utilizes plant fibers as replacements of glass fibers in polymeric composites, thereby reducing the petroleum consumed by glass fiber production. Numerous articles

Correspondence to: Wonsuk Kim; e-mail: wskim@umich.edu

Contract grant sponsor: National Science Foundation; contract grant number: 0800254

DOI 10.1002/pc.21169

Published online in Wiley Online Library (wileyonlinelibrary.com). (C) 2011 Society of Plastics Engineers are available pursuing the use of plant fiber based composites for load bearing applications that are currently made with glass fiber composites, including work on material arrangement, testing and analysis [1-11] and processing techniques [12-14] to achieve high stiffness and strength. Although not renewable, nanofillers based on montmorillonite are obtained from natural sources and, like the plant based fiber reinforcements, can also displace energy intensive fiberglass in polymer composite applications. In automotive applications, both natural fibers and nanofillers can offer weight reductions over conventional composites that can translate to improved fuel economy.

This article concerns the capacity of various bio-, nano-, and microcellular composite systems to dissipate impact energy in safety settings through internal mechanical processes including composite failure mechanisms and cellular collapse of micro-voids and/or vascular plant fibers. This functionality is to some degree orthogonal to load bearing capability since, in general, compared to load-bearing requirements, energy-dissipation characteristics benefit from low strain-rate dependence, reasonably low elastic modulus, and an extended post yield region of high deformation. A review of the presently available literature indicates that very little work has been done in this regard for biomaterials. The few available works either simply indicate the potential of these materials for crash applications [15] or have begun to look at rate dependent constitutive properties [16].

Strain-rate dependence or rate hardening, refers to the tendency of a material's yield stress to increase and failure strain to decrease when loaded at high rate. Polymeric materials, especially glass fiber reinforced type, are typically highly strain-rate dependent which reduces their ability to dissipate impact energy at low stress. Low strain-rate dependence has been observed in certain types of biocomposites suggesting they are appropriate for applications where the material is nominally low load bearing (since these materials typically have lower nomi- 
nal stiffness than glass fiber composite), but must be able to dissipate impact energy for safety purposes. For example, there are many automotive applications whose function is energy dissipation, ranging from exterior body panels for pedestrian impact protection to interior impact absorbing material behind pillars for head impact.

In this article, the constitutive behavior at high strain rate of plant fiber-reinforced composites, as well as nanoreinforced composites, infused with microcellular voids is studied. Compressive stress-strain curves of the various composite samples are experimentally obtained at high strain rates using a split Hopkinson pressure bar apparatus, which is described in the following section. Based on the experimental results, an interesting feature concerning muted strain-rate dependence in plant fiber composites and the effect of nanoclay on the strain-rate dependence are discussed. Also, biocomposites and nanocomposites with infused microcellular voids are treated. Finally, microscopic inspections of untested and tested specimens are given and the roles of microcellular voids and the plant fibers' natural vasculature on the strain-rate dependence are discussed.

\section{HOPKINSON TESTING}

Strain-rate dependence at high rates of strain is determined here by the Hopkinson method. The technique, which is described in detail in Al-Mousawi et al. [17], involves transmission of high rate strain through a small disk-shaped specimen placed between long input and output cylindrical bars (see Fig. 1). The input bar is impacted by a short striker bar, inducing longitudinal waves to propagate and pass through the specimen and into the output bar. Small strains produced in the bars are measured and used to infer the stress-strain behavior of the specimen via techniques of elastic wave propagation. The maximum strain rate induced in the material is controlled by the mass and speed of the striker. The standard Hopkinson apparatus and technique are limited to relatively stiff materials and rates above about 400 strain $\times \mathrm{s}^{-1}$, as soft materials and low impact speeds reduce the energy transmitted through the specimen resulting in a weak strain signal in the output bar. Here, materials having modulus as low as about $0.5 \mathrm{GPa}$ have been tested. Because of the somewhat low material modulus, special care was required in the tests concerning selecting and locating strain gages on the bars, lining up input and output signals and reducing noise. Half Wheatstone bridge circuits were used to amplify strain gage signals.

Bulk materials for the Hopkinson tests have been manufactured by various methods, as described in the following sections, and cut into solid circular discs by mechanical punching or a water jet process. All discs used in the tests had nominal dimensions of $12.7 \mathrm{~mm}$ in diameter and 3-3.4 $\mathrm{mm}$ in thickness.

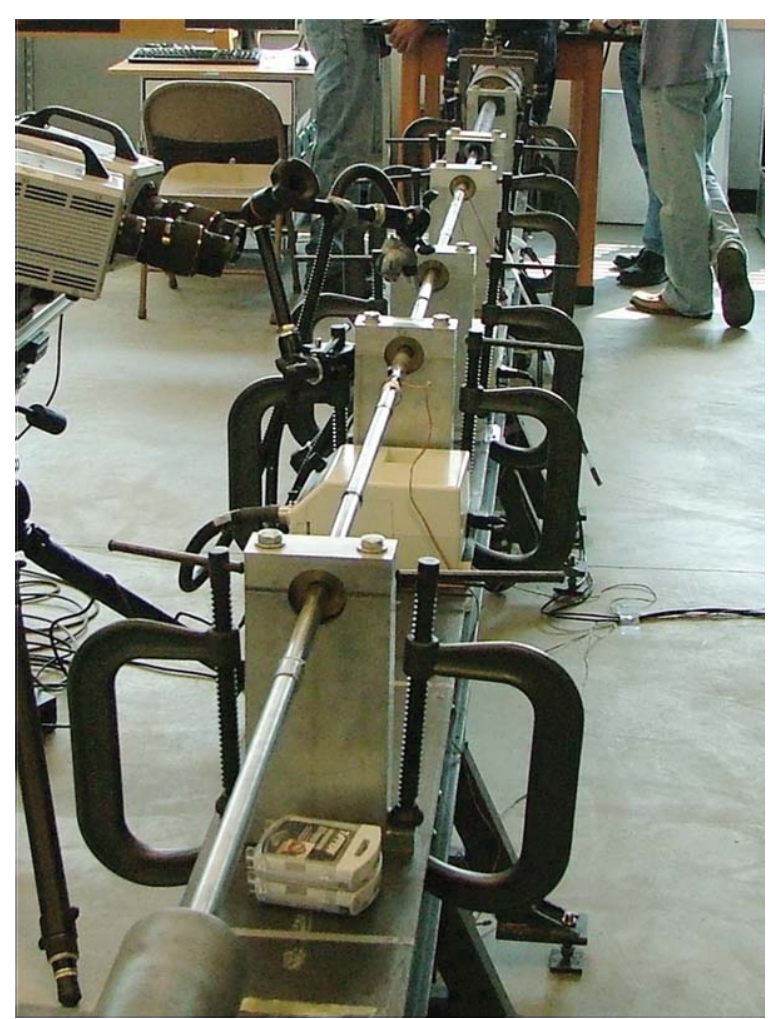

FIG. 1. Split Hopkinson pressure bar apparatus. [Color figure can be viewed in the online issue, which is available at wileyonlinelibrary.com.]

\section{RESULTS AND DISCUSSION}

\section{Effects of Plant Fibers on the Strain-Rate Dependence}

Results are given in this section of tests on two conventional materials (polypropylene and glass fiber/vinyl ester composite) and two biocomposite materials (sisal fiber/polypropylene and hemp mat/vinyl ester).

The effects of plant fibers on strain-rate dependence are shown in both thermoplastic and thermoset composite systems. In the thermoplastic system case, unreinforced polypropylene is compared with $30 \%$ sisal fiber-reinforced polypropylene. The sisal fiber-reinforced composite was prepared by standard extrusion compounding with a proprietary formulation. Test specimens for static tests were prepared directly by standard injection molding. For high rate Hopkinson measurements, specimens were cut from $102 \mathrm{~mm} \times 102 \mathrm{~mm}$ injection molded plaques of $3 \mathrm{~mm}$ thickness. For thermoset systems, glass fiber/vinyl ester, and hemp mat/vinyl ester composites are compared. Both composite systems were prepared on standard sheet molding compounding equipment. Compounded materials were compression molded after a $24-$ to 48 -h maturation period to $305 \mathrm{~mm} \times 305 \mathrm{~mm}$ plaques of $3 \mathrm{~mm}$ thickness. Molded plaques were prepared to desired dimensions by bandsaw/router and by water jet for static and high rate Hopkinson tests, respectively. 


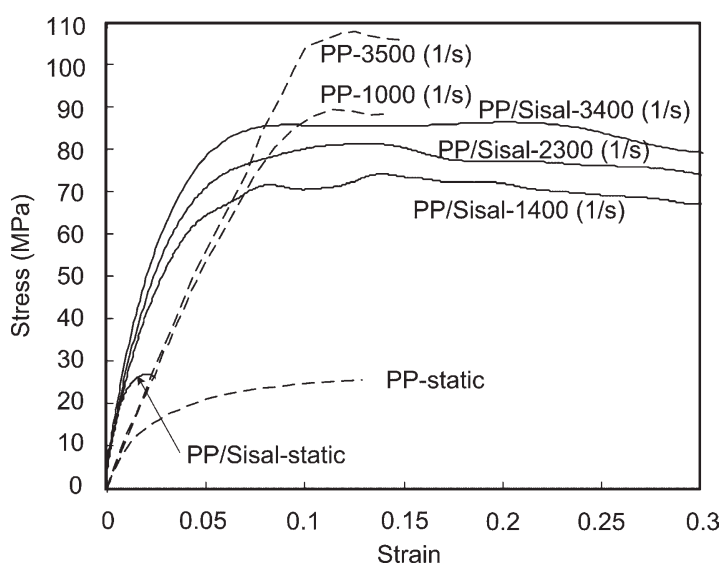

FIG. 2. Stress-strain curves of neat polypropylene (PP) and polypropylene with sisal fibers $(\mathrm{PP} / \mathrm{Sisal})$ at various strain rates.

Shown in Fig. 2 are stress-strain measurements of neat polypropylene (PP) and sisal fiber/polypropylene composite (PP/Sisal) specimens at various rates. Tensile static behavior is also shown for comparison. It should be noted that in these tests the end of a curve does not indicate material failure, but rather that the test has ceased because the impact energy has dissipated. Also, the elastic modulus from these tests should be regarded as approximate since its precise measurement by the Hopkinson method is not always reliable and should be confirmed by other measurements $[17,18]$. Typically, the modulus is far less affected by loading rate than flow stress in this class of materials. In all the present cases, the Hopkinson moduli are seen to line up with static measurements and so are felt to be reasonably accurate. Lastly, precise control of the strain rate induced in the specimens is difficult to achieve in these tests, and is partly a function of the material properties of the specimen, so the strain rates are not exactly the same for every material case. Moreover, the strain rate varies during a test. The strain rate value presented in this article is the maximum strain rate induced during the test.

Because a clearly defined yield point is not discernable in these materials, rate hardening comparisons are made using the maximum stress. Comparing the static response of neat polypropylene to its behavior when loaded at a strain rate of 1,000 strain $\times \mathrm{s}^{-1}$ reveals a rate hardening of about $260 \%$. This means if an impact event induces PP to deform at 1,000 strain $\times \mathrm{s}^{-1}$, the material reaches a maximum stress of about $90 \mathrm{MPa}$ rather than its static value of about $25 \mathrm{MPa}$. The maximum stresses occur at about the same strain value. In the 1,000 strain $\times \mathrm{s}^{-1}$ case, the accompanying softening (large deformation) occurring around yield is thereby delayed to high stress. This behavior reduces the time it takes for the PP material to decrease the impacting body's kinetic energy to zero and so is accompanied by a high peak contact force and stress in the impacting body.

In contrast, PP with $30 \%$ sisal fibers (PP/Sisal) in Fig. 2 shows less rate hardening than neat PP. Specifically,

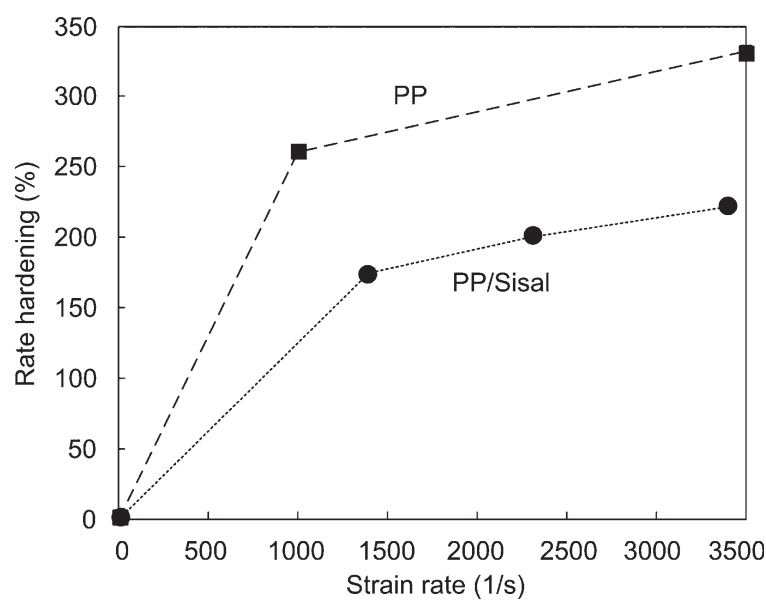

FIG. 3. Rate hardening of neat polypropylene (PP) and polypropylene with sisal fibers (PP/Sisal).

rate hardening of only $174 \%$ occurs in the PP/Sisal composite at 1,400 strain $\times \mathrm{s}^{-1}$ whereas $260 \%$ occurs in the neat PP sample at 1,000 strain $\times \mathrm{s}^{-1}$. A comparison of the rate hardening of the two materials is shown in Fig. 3. It is clear that the addition of sisal fibers to PP reduces rate dependence. This effect has also been observed to some degree in other biocomposites. For example, Fig. 4 shows the measured stress-strain responses of chopped glass and hemp fiber-reinforced vinyl ester samples at various strain rates. Both materials exhibit rate hardening, though the hemp fiber curves also display a lack of clearly defined yield points and possess smooth transition into high strain regions, characteristics beneficial for energy absorption. A similar feature has been observed during static testing in [19] where the authors observe cellulose failing in a "graceful manner with slow and stable crack growth." Because static data is not available for these materials, rate hardening can be compared by the normalized percent increase in peak stress over two rates:

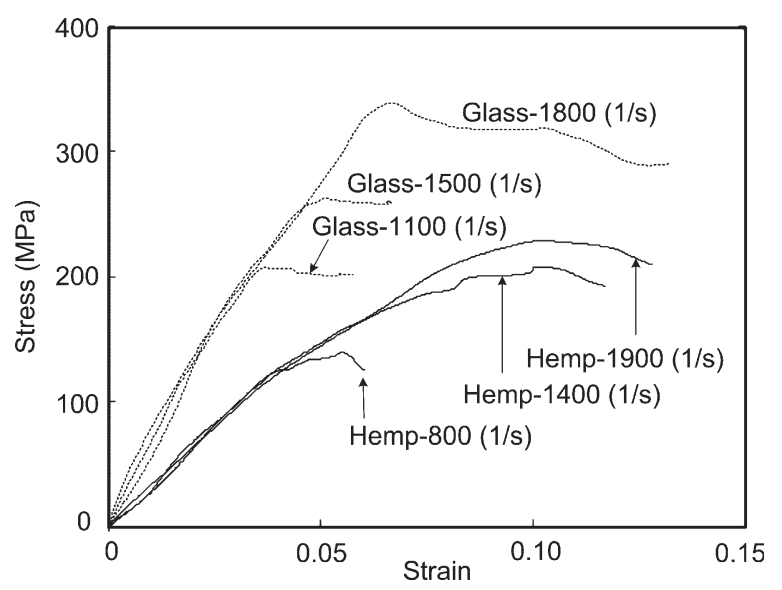

FIG. 4. Stress-strain curves at various strain rates of two chopped fiber composites: glass/vinyl ester (Glass) and hemp mat/vinyl ester (Hemp). 


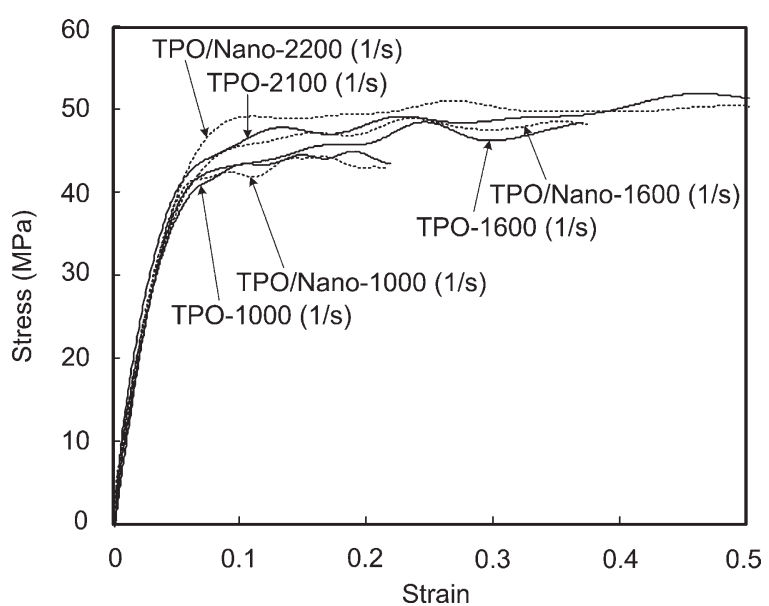

FIG. 5. Stress-strain curves of thermoplastic olefin (TPO) and thermoplastic olefin with nanoclays (TPO/Nano) at various strain rates.

$$
P=\frac{\sigma_{2}-\sigma_{1}}{\sigma_{1}\left(\dot{\varepsilon}_{2}-\dot{\varepsilon}_{1}\right)} \times 100
$$

where $\sigma_{i}$ is the peak stress on the $\dot{\varepsilon}_{i}$ strain-rate curve. Applying this to the three pairs of strain-rate curves for each material, then averaging, gives $P_{\text {avg }}=0.083 \% /(1 / \mathrm{s})$ for the glass composite (i.e., percent increase in peak stress per strain rate) and $P_{\text {avg }}=0.053 \% /(1 / \mathrm{s})$ for hemp. Thus, on average, rate hardening is $57 \%$ higher in the glass composite than in the hemp.

\section{Effects of Nanoclay on the Strain-Rate Dependence}

So-called nanoclays are layered silicates that have been organically modified by cation exchange of the naturally occurring sodium ion with an alkyl ammonium ion. The exchange renders the platelet-shaped nano-particles compatible with a polymer resin matrix. A nanocomposite material containing nanoclay specially dispersed in thermoplastic olefin (TPO/Nano) has been manufactured and tested using the Hopkinson apparatus. Thermoplastic olefin is polypropylene modified with rubber, which would therefore be expected to possess muted rate dependence. The TPO/Nano material evaluated here was prepared from a commercially available nanocomposite masterbatch from Nanocor (Hoffman Estates, IL) let down to a nanoclay loading of $4 \%$ by weight. Test specimens were cut from standard injection molded plaques.

In Fig. 5, the ability of nanoclay filler to mute rate dependence is studied by comparing the response of TPO to TPO/Nano at various rates. The TPO curves indicate that the rubber modified material has greatly muted rate dependence compared to the PP homopolymer shown in Fig. 2. Furthermore, the TPO/Nano curves indicate that the addition of nanoclay filler to the rubber modified formulation offers no enhancement or degradation of the stiffness and rate dependence.
Effects of Microcellular Voids on the Strain-Rate Dependence

Samples with micron sized voids were also investigated at high strain rate to study the effect of microstructure on rate dependence. The microcellular structure in these samples was created by using Trexel's physical foaming process, known as MuCell. This process consists of injecting a minute amount of supercritical fluid directly into the polymer melt during screw recovery, forming a one-phase solution, and injecting the mixture into a mold tool. During the mold filling, the material shot size is reduced by a desired amount and the dissolved gas comes out of solution to create micron-sized voids. The foaming causes the reduced amount of material to fully fill the volume of the tool. In order for the process to yield materials with uniform microcellular structure, a reinforcing filler material is recommended. Here two separate reinforcements are used-sisal fibers and nanoclay.

A biocomposite with $30 \%$ sisal fibers in PP and containing $10 \%$ microcellular voids (PP/Sisal, MuCell) was molded by the MuCell process with $0.66 \%$ supercritical $\mathrm{N}_{2}$ by weight. Note that by the description " $10 \%$ microcellular voids," it is meant that the weight of the microcellular composite is $10 \%$ less than the corresponding solid composite. Static data were measured on molded test bar specimens, while Hopkinson specimens were cut from molded samples. As shown in Fig. 6, the material displays minor rate hardening through strain rates of 2,400 strain $\times \mathrm{s}^{-1}$ when compared to its static response. In Fig. 7, the PP/Sisal with MuCell at 2,400 strain $\times \mathrm{s}^{-1}$ case is compared to PP/Sisal (without MuCell, i.e., solid) at 2,300 strain $\times \mathrm{s}^{-1}$. Although the addition of plant fibers to PP reduces the rate dependence over neat PP, the addition of microcellular voids has a further benefit to energy dissipation. It is seen in the figure that PP/Sisal with MuCell smoothly transitions through its apparent yield point into large deformation, and continues in the high deformation region with gradually increasing stress.

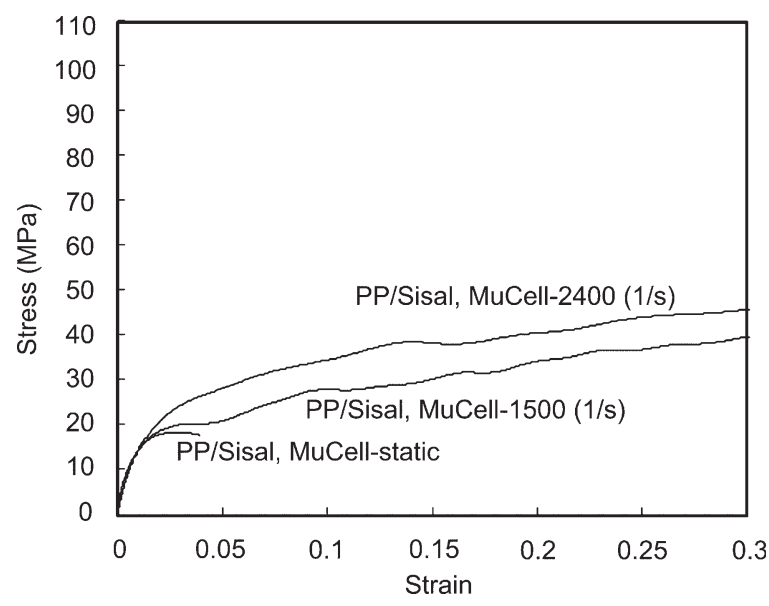

FIG. 6. Stress-strain curves of polypropylene with sisal fibers and microcellular voids (PP/Sisal, MuCell) at various strain rates. 


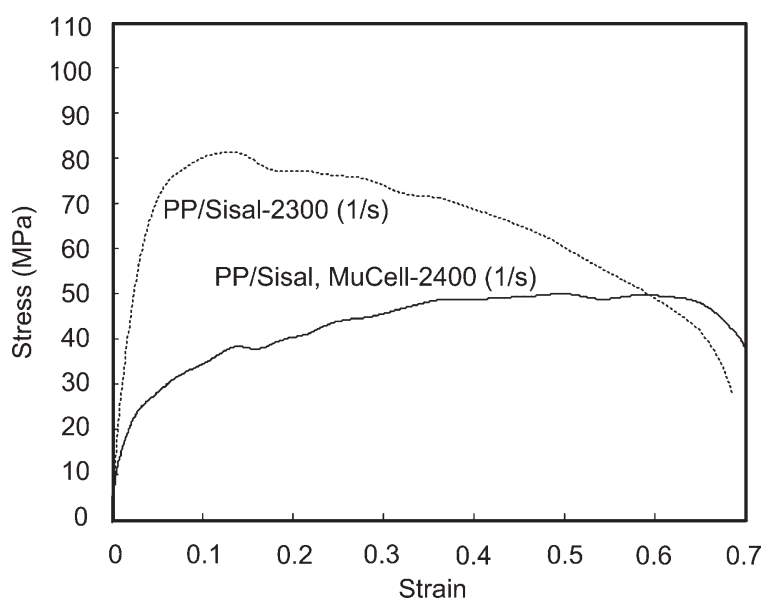

FIG. 7. Stress-strain curves of sisal fiber/polypropylene composite materials with/without microcellular voids at high strain rates.

These characteristics are very positive for impact safety applications since impact energy dissipation starts at low stress and proceeds continuously through high strain regions without catastrophic failure.

To investigate these behaviors further, TPO/Nano materials with microcellular voids have also been manufactured and tested. The samples used in the current study were processed by MuCell using 3\% supercritical $\mathrm{CO}_{2}$ by weight. Samples having 6 and $12 \%$ microcellular voids were produced. High rate data were measured on specimens that were cut from MuCell molded plaques. Shown in Fig. 8 are stress-strain curves at $\sim 2,000$ strain $\times \mathrm{s}^{-1}$. The dotted line denotes TPO/Nano with 6\% MuCell which exhibits a traditional constitutive characteristic similar to the solid line obtained in the TPO/Nano case. However, in the case of $12 \%$ MuCell (shown by the dashed line), the behavior of gradually increasing stress through the large deformation region observed in Fig. 7 is

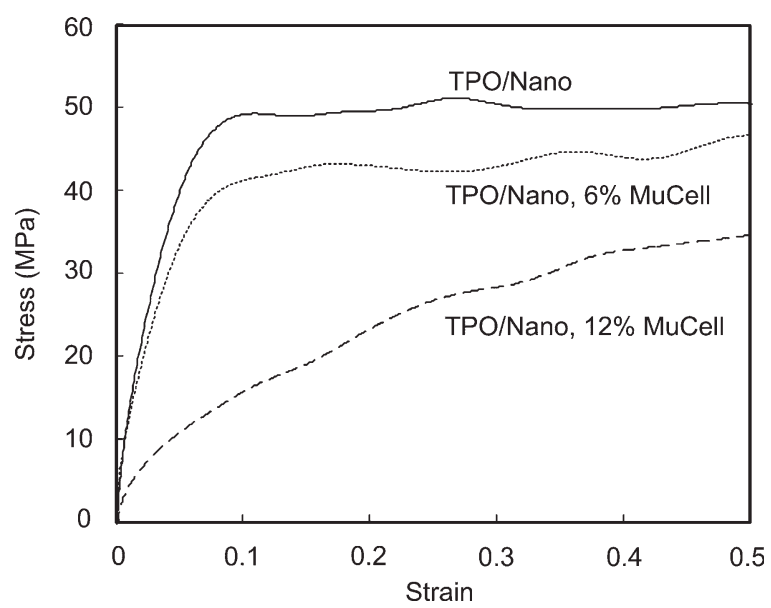

FIG. 8. Stress-strain curves at $\sim 2,000$ strain $\times \mathrm{s}^{-1}$ of thermoplastic olefin/nanoclay composite materials with various amounts of microcellular voids: no MuCell (solid line), 6\% MuCell (dotted line), and 12\% MuCell (dashed line).

again seen. It appears that while rubber modifier, plant fibers and infused microcellular voids all greatly suppress rate hardening, the infused voids most strongly induce smooth foam-like behavior at high rate. Plant fibers also produce this behavior, as can be seen most clearly in the sisal fiber case (see Fig. 2), but to a lesser extent.

\section{Microscopic Inspection}

Although most fiber-reinforced polymeric composites do not yield in the traditional metallic sense of large plastic deformation, they can exhibit transition to large deformation response in which very large energy is dissipated with little increase in load. This process can be generally characterized by matrix cracking, fiber transverse fracture and axial splitting, fiber pull-out from the matrix, etc. In the case of the natural fibers investigated in this article,

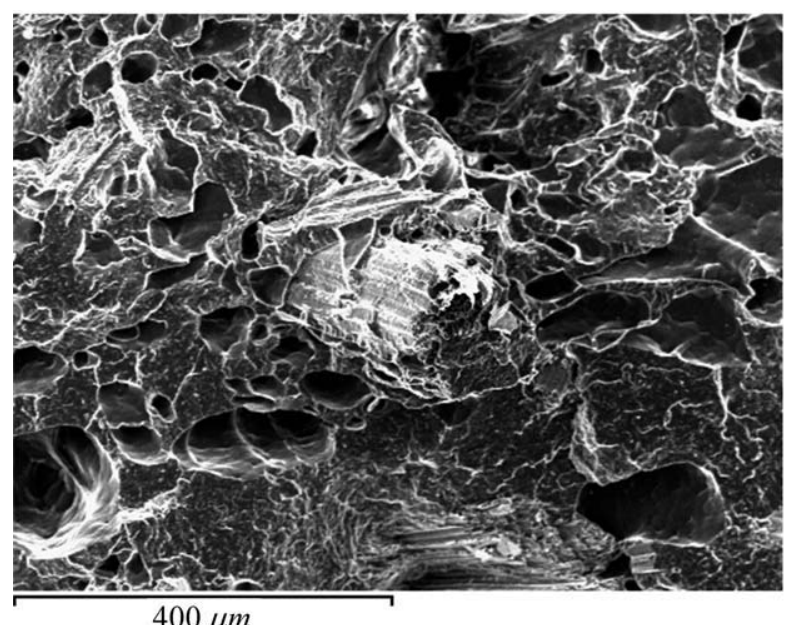

(a)

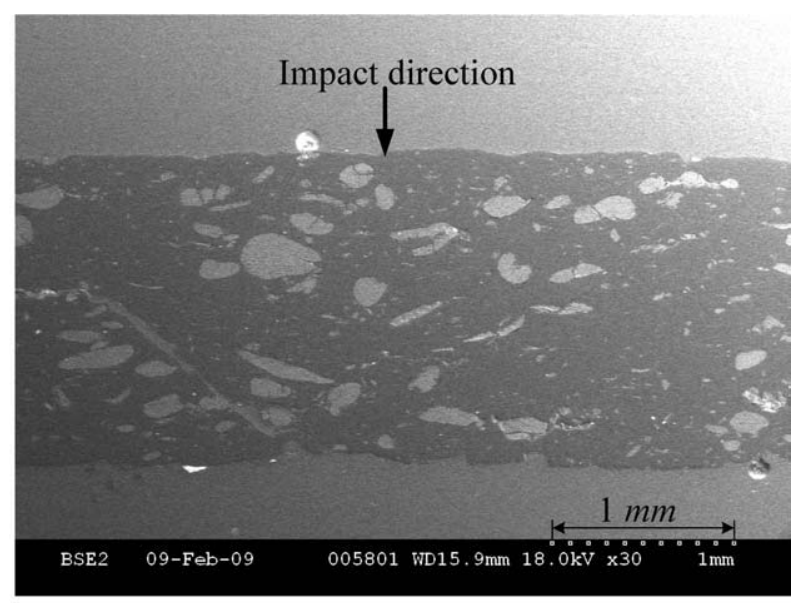

(b)

FIG. 9. Microcellular polypropylene containing sisal fibers: (a) untested sample, (b) sample impacted at rate of 2,400 strain $\times \mathrm{s}^{-1}$. 


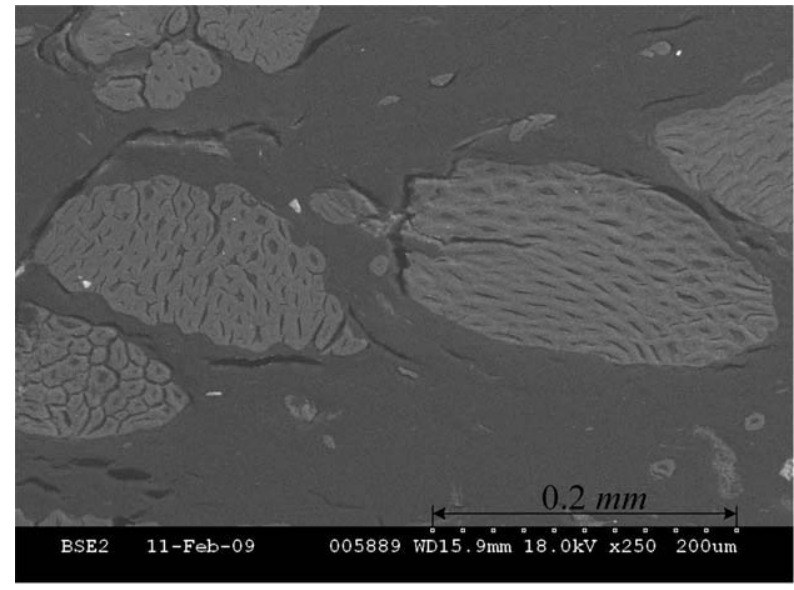

(a)

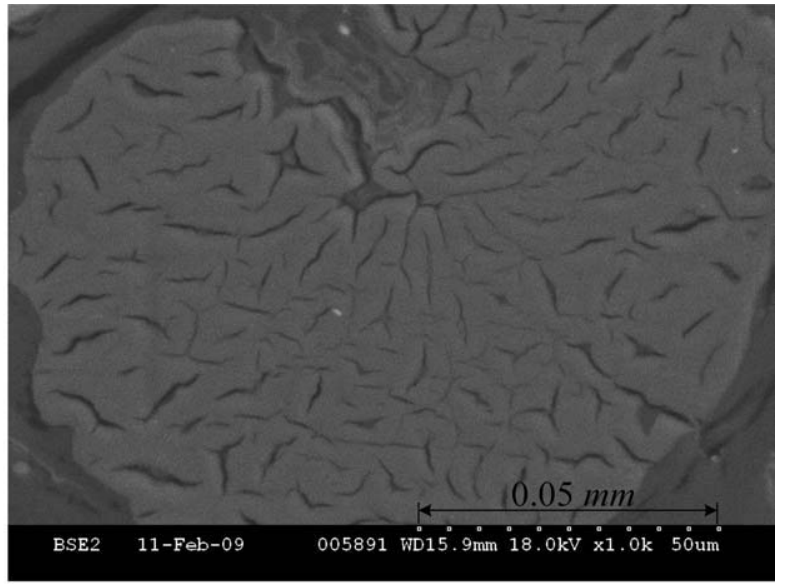

(b)

FIG. 10. Microcellular polypropylene containing sisal fibers impacted at rate of 2,400 strain $\times \mathrm{s}^{-1}$ : (a) fiber cross-sections at lower magnification, (b) fiber cross-section at higher magnification.

the vasculature of the plant materials may add another energy dissipation mode to the overall process. To study this feature as well as the behavior of the infused microcellular voids, microscopic inspections have been performed.

Figure 9a shows a portion of a typical untested microcellular PP/sisal specimen revealing fibers surrounded by voids and PP. Overall, the specimen contains 30\% sisal fibers; the infusion of microcellular voids resulted in a $10 \%$ weight reduction and an average void size of about $100 \mu \mathrm{m}$. Figure $9 \mathrm{~b}$ shows a specimen after 2,400 strain $\times$ $\mathrm{s}^{-1}$ impact. The material undergoes tremendous crush from its original thickness of $3 \mathrm{~mm}$ to about $1.7 \mathrm{~mm}$. Figure 10 shows two magnifications of the tested specimen revealing the post test vasculature of the sisal fibers. In some regions fiber void space is closed, likely due to the impact, and in other regions the fibers have not closed due to the load paths or because they have been filled by
PP during the manufacture. These images suggest a possible mechanism for the muted rate dependency seen in the plant fiber cases in Figs. 2 and 4.

Untested and tested impact specimens of TPO/Nano with $12 \%$ MuCell have also been microscopically inspected. Figure 11a shows a typical distribution of micro cells in untested TPO with exfoliated nanoclay. The microcellular foaming process yields a region of voids in the center of the specimen and a "skin" layer, or nonfoamed region, on the surface. Here, processing was controlled such that the skin dimension and the length of a typical oblong void are roughly $650 \mu \mathrm{m}$. Figure $11 \mathrm{~b}$ of a sample after 1,200 strain $\times \mathrm{s}^{-1}$ impact shows collapsed voids and a generally maintained overall structure. It is thought that the process of void collapse is the source of the smooth transition to large strain regions observed in Figs. 6 and 8.

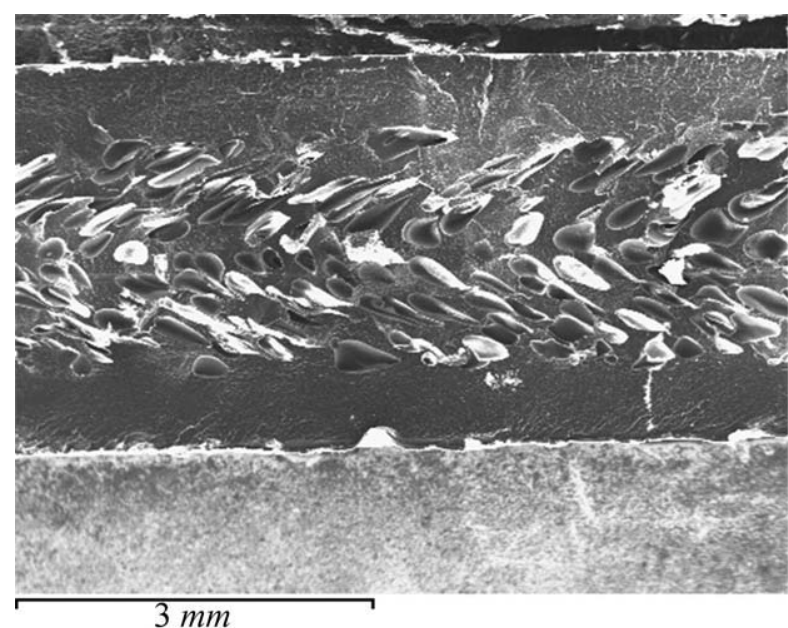

(a)

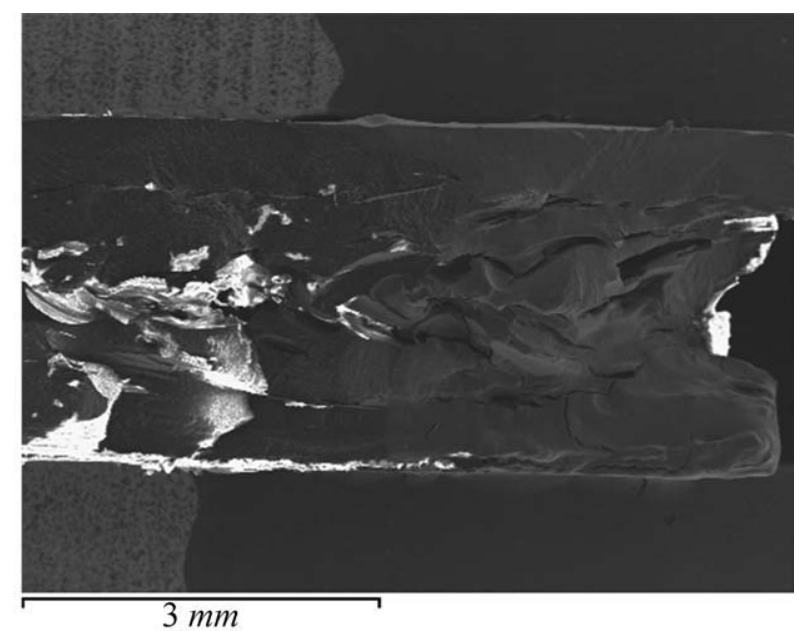

(b)

FIG. 11. Microcellular TPO with exfoliated nanoclay: (a) untested sample, (b) sample impacted at rate of 1,200 strain $\times \mathrm{s}^{-1}$. 


\section{CONCLUSIONS}

Biocomposites and nanocomposites consisting of plant fibers and nanoclays, respectively, embedded in binding resin and infused with microcellular voids have been investigated. These materials have been found to possess uniquely advantageous energy dissipation characteristics in a rigid, lightweight form, offering potential for a great range of impact safety applications. Preliminary trials have shown that the new formulations dissipate impact energy in a gradual, relatively rate insensitive manner, primarily due to infused voids. Another possible dissipation mechanism has been observed related to deformation of the vasculature of the natural fibers, though the materials are complex and the exact mechanisms are not fully understood at this point. Further studies are necessary to completely describe the mechanisms at work and design optimum formulations.

\section{ACKNOWLEDGMENTS}

This paper is based upon work supported by the National Science Foundation under grant number 0800254. The support is gratefully acknowledged. Any opinions, findings, and conclusions or recommendations expressed in this material are those of the authors and do not necessarily reflect the views of the National Science Foundation. The authors thank Dr. Terry Ostrom for conducting some of the microscopy. The authors also acknowledge Nathan Stewart and Mariam Farhat for their work in preparing some experimental results.

\section{REFERENCES}

1. W.D. Brouwer, SAMPE J., 36, 18 (2000).

2. E.S. De Medeiros, J.A.M. Agnelli, K. Joseph, L.H. De Carvalho, and L.H.C. Mattoso, Polym. Compos., 26, 1 (2005).
3. M. Hughes, C.A.S. Hill, and J.R.B. Hague, J. Mater. Sci., 37, 4669 (2002).

4. S. Wong, R.A. Shanks, and A. Hodzic, Macromol. Mater. Eng., 289, 447 (2004).

5. H. Cabral, M. Cisneros, J.M. Kenny, A. Vazquez, and C.R. Bernal, J. Compos. Mater., 39, 51 (2005).

6. E. Rodriguez, R. Petrucci, D. Puglia, J.M. Kenny, and A. Vazquez, J. Compos. Mater., 39, 265 (2005).

7. I. Taha and G. Ziegmann, J. Compos. Mater., 40, 1933 (2006).

8. L. Onal and Y. Karaduman, J. Compos. Mater., 43, 1751 (2009)

9. J. Biagiotti, S. Fiori, L. Torre, M.A. Lopez-Manchado, and J.M. Kenny, Polym. Compos., 25, 26 (2004).

10. P.-O. Hagstrand and K. Oksman, Polym. Compos., 22, 568 (2001).

11. V.N. Hristov, S. Vasileva, M. Krumova, R. Lach, and G.H. Michler, Polym. Compos., 25, 521 (2004).

12. A. Keller, Compos. Sci. Technol., 63, 1307 (2003).

13. M.A. Khan, C. Kopp, and G. Hinrichsen, J. Reinforce. Plast. Compos., 20, 1414 (2001).

14. L.Y. Mwaikambo and M.P. Ansell, J. Appl. Polym. Sci., 84, 2222 (2002).

15. M. Pervaiz and M.M. Sain, Macromol. Mater. Eng., 288, 553 (2003).

16. J. George, S. Thomas, and S.S. Bhagawan, J. Thermoplast. Compos. Mater., 12, 443 (1999).

17. M.M. Al-Mousawi, S.R. Reid, and W.F. Deans, J. Mech. Eng. Sci., 211, 273 (1997).

18. T. Nicholas, Exp. Mech., 21, 177 (1981).

19. I.M. Low, M. Mcgrath, D. Lawrence, P. Schmidt, J. Lane, B.A. Latella, and K.S. Sim, Compos. A Appl. Sci. Manufact., 38, 963 (2007). 